\title{
Microzonificación sísmica en las aldeas: Jacaleapa, El Tablón y La Cañada, Tegucigalpa D.C., Honduras (2017-2018)
}

\author{
Seismic Microzoning in the Villages: Jacaleapa, El Tablón and La Cañada, Tegucigalpa \\ D.C., Honduras (2017-2018) \\ DOI 10.5377pc.v0i16.8099
}

Gerardo Pineda

José David Cáceres ${ }^{2}$

\section{RESUMEN}

Esta investigación muestra datos fundamentales para el diseño de estructuras ya que se obtuvieron puntos de frecuencia, amplitudes relativas, alturas definidas del basamento rocoso, tipo de suelos y rocas, velocidades de ondas que caracterizan los estratos, así como una cartografía temática por medio de mapas, que servirán como instrumento para realizar una buena planificación urbana y obtener el fin de ordenar el territorio.

El objetivo de esta investigación es el de establecer una microzonificación sísmica, que consiste en identificar y caracterizar unidades litológicas, generalmente suelos cuya respuesta dinámica frente a terremotos son semejantes. Además de estas unidades se incluyen los efectos inducidos (fallas, licuefacción, etc.) y se valora su peligrosidad. Los mapas resultantes, o mapas de microzonación, se presentan en una base cartográfica útil para fines de edificación y planificación urbana.

Para obtener Los ensayos 0 estudios realizados en esta investigación se recabo información nacional y se realizaron diferentes mapas temáticos con el propósito de conseguir una geología a detalle del tipo de suelo y roca que conforma el sitio, pruebas de Refracción Sísmica, Resistividad Eléctrica, y Períodos del suelo con el acelerógrafo, así como la topografía que ayudo a generar el Modelo Digital de Elevaciones (MDE), mismo que ayudo a identificar zonas de deslizamiento en la zona de estudio.

\footnotetext{
${ }^{1}$ Estudiante de Maestría en Demografía y Desarrollo, Facultad de Ciencias Sociales, UNAH: gerardog_316@hotmail.com

2 Asesor, Ingeniero Ambiental, Máster en Tecnología de la Información Geográfica, Profesor Titular II del Departamento de Ciencias y Tecnologías de la Información Geográfica, Coordinador Académico de la Licenciatura en Ciencias y Tecnologías de la Información Geográfica, Profesor Titular de la Maestría en Ordenamiento y Gestión del Territorio. (Todo lo anterior en la UNAH)
} 
Son dos microzonas que se establecieron en este estudio en base al período fundamental del suelo, la zona I que va desde 1.25 a 1.79 segundos y la zona II que va desde 0.43 a 0.80 segundos, para la zona I las edificaciones en riesgo son las que poseen una altura promedio correspondiente arriba de los diez pisos. En la zona dos las edificaciones de 3 a 4 pisos.

Palabras clave: Microzonificación sísmica, efecto de sitio, efecto de resonancia.

\section{ABSTRACT}

This research shows fundamental data for the design of structures since points of frequency, relative amplitudes, defined heights of the rocky basement, type of soils and rocks, wave velocities that characterize the strata, as well as a thematic cartography by means of maps were obtained, which will serve as an instrument to carry out a good urban planning and obtain the order of ordering the territory.

The objective of this research is to establish a seismic microzonation that consists in identifying and characterizing lithological units, generally soils whose dynamic response to earthquakes are similar. In addition to these units, the induced effects are included (faults, liquefaction, etc.) and their dangerousness is assessed. The resulting maps, or microzonation maps, are presented in a cartographic base useful for urban planning and building purposes.

To obtain the essays or studies carried out in this research, national information was collected and different thematic maps were made in order to obtain a detailed geology of the type of soil and rock that makes up the site, Seismic Refraction, Electrical Resistivity, and Periods of the soil with the accelerograph, as well as the topography that helped to generate the Digital Elevation Model (DEM), which helped to identify landslide zones in the study area.

There are two microzones that were established in this study based on the fundamental period of the soil, zone I that goes from 1.25 to 1.79 seconds and zone II that goes from 0.43 to 0.80 seconds, for zone I the buildings at risk are those that they have a corresponding average height above ten floors. In zone two the buildings of 3 to 4 floors.

Keywords: seismic microzoning, site effect, resonance effect. 


\section{INTRODUCCIÓN}

Honduras se encuentra dentro del Cinturón o Anillo de Fuego (Ring of Fire), que se caracteriza por concentrar algunas de las zonas de subducción más importante del mundo, siendo más específico dentro de la convergencia de la Placa de Coco, Placa de Norteamérica, Placa del Caribe y la Placa de Nazca. Los movimientos sísmicos registrados en el país muestran que la mayor actividad sísmica se ha dado en la dirección Noroeste y Suroeste de Honduras.

La zona de estudio está ubicada en el Distrito Central, dicha zona está caracterizada como una región de amenaza media de sismicidad. Los datos sísmicos existentes para Honduras se clasifican en dos periodos; el primero es una descripción histórica de los terremotos que ocurrieron desde 1539-1898 (periodo pre-instrumental), el segundo es desde 1898-2011, que es el periodo instrumental. Según datos del U.S Geological Survey (USGS) en Honduras y sus regiones aledañas se han presentado, desde 1648 hasta 2009, un total de 4,276 sismos de baja, media y alta intensidad.

Mediante los ensayos realizados en esta investigación que fueron los de Resistividad Eléctrica, Refracción Sísmica, Topografía, Geología y puntos tomados con el Acelerógrafo Basalt, se puede conocer el peligro sísmico de una región y saber la probabilidad de que se produzca en ella movimientos sísmicos de una cierta importancia en un plazo determinado. También se obtienen períodos fundamentales del suelo, amplificación del movimiento del terreno, espectros de respuestas para diseño y seguridad de las estructuras, todos estos datos son necesarios para conocer el denominado "Efecto de Sitio".

El Efecto de Sitio Se define como la influencia de las propiedades geotécnicas, geológicas y topográficas de los estratos más superficiales de la corteza terrestre en las características de los movimientos sísmicos esperados para un sitio. La presencia de terrenos, considerados geotécnicamente como blandos, próximos a la superficie del terreno produce un aumento considerable de los daños generados por terremotos en las infraestructuras situadas sobre ellos, al condicionar la amplificación de las ondas sísmicas en un rango de periodos de vibración (períodos altos) de los materiales, que coincide con el periodo de vibración de las estructuras.

Cuando el período de vibración de la estructura y el período del suelo donde se apoya coinciden, los efectos sísmicos se amplifican, y es casi seguro que se derrumbe la estructura, a este fenómeno se le conoce como resonancia; Por lo que puede evitar- 
se, construyendo estructuras con períodos que no coincidan con el del suelo.

Las amenazas sísmicas traen consigo daños a la economía y esto a su vez trae una agravación de la calidad de vida de toda la población que reside en zonas vulnerables. El espacio superficial que comprende las Residenciales Venecia, El Tablón, Paris y el Proyecto Althia tiene un área aproximada de $2.71 \mathrm{Km}^{2}$, la población actual va desde niños hasta adultos mayores.

En la actualidad se han dado problemas de deslizamiento debido a las fuertes lluvias que periódicamente afectan el país esto trae consigo movimiento de terreno el cual afecta las estructuras existentes, estos sectores están catalogadas como zonas vulnerables por COPECO, el hecho de que estos terrenos se consideren como vulnerables es un factor a tomar en cuenta para la realización de demarcaciones urbanas, y es punto de partida para empezar a ordenar el territorio, y así empezar a construir con normas de calidad y seguridad.

En mayo 22 del año 2012, una serie de daños a la infraestructura fue causada por las precipitaciones que afectan a Tegucigalpa año tras año, la precipitación hace que se activen las diferentes fallas geológicas que se encuentran ubicadas en toda la zona de estudio provocando derrumbes y daños a las estructuras existentes por diferentes tipos de vibraciones en el suelo.

En el año 2012 parte del pavimento de la calle de acceso a la Residencial Venecia, ubicada a un costado del anillo periférico, cerca de la Universidad Tecnológica de Honduras (UTH), cedió debido a la humedad que labró la tierra provocando un hundimiento y un gran agujero en el pavimento. Los pobladores de Residencial Paris han denunciado caídas de muros y hasta han afirmado la caída de "un cerro".

Es por esto que se busca una zonificación sísmica que consiste en dividir una región en porciones, en cada una de ellas se especifican parámetros constantes de diseño sísmico. El movimiento de la superficie en un terreno dado puede diferir radicalmente del que se tiene en la roca base, debido a alteraciones de las ondas causadas por efectos geológicos, topográficos y de rigidez del subsuelo. La importancia de estas alteraciones, llamadas en términos generales efectos locales, ha sido considerada cada vez más en los años recientes y ha conducido a la necesidad de realizar estudios de microzonificación de las áreas de asentamientos humanos, para detectar aquellas zonas que presentan problemas especiales.

Los estudios de microzonificación sísmica consisten en estudios multidisciplinarios. 
Se consideran los efectos que un sismo tendría en la zona, tomando en cuenta los efectos de sitio y microzonas sísmicas. Los resultados finales quedan plasmados en un mapa de microzonificación sísmica, donde el área de estudio es dividida en sectores de diferente riesgo potencial. Con esto se tendrá la información necesaria para inferir los efectos de un terremoto sobre las edificaciones, dentro de un área urbanizada.

\section{METODOLOGÍA}

\section{Caracterización Preliminar}

En esta etapa de la investigación se comenzó enviando diferentes solicitudes de apoyo a la Universidad Nacional Autónoma de Honduras UNAH, específicamente al Instituto Hondureño de Ciencias de la Tierra IHCIT y a la Comisión Permanente de Contingencias COPECO, dichas instituciones poseen el equipo y personal para realizar los ensayos que se han realizado para la obtención de resultados de esta investigación; también se analizaron las solicitudes enviadas por los vecinos y urbanizadores que se quejaron por la situación de muchas viviendas afectadas por deslizamientos y hundimientos de las estructuras.

Se empezó a analizar y recabar información nacional existente, en donde cabe recalcar que lo más utilizado fueron las capas o shapes, necesarias para georreferenciar la zona de estudio y la realización de los mapas temáticos en software como ArcGis, con dichas capas obtuvimos los mapas necesarios para saber qué tipo de zona se estudiaba. Los mapas realizados con información nacional y mediante fuente propia fueron los siguientes:

- Ubicación de la Zona de Estudio

- Amenazas Símicas de Honduras.

- Mapa Geológico del País (IGN).

- Mapa Geológico del País (LOTTI)

- Mapa de Inundaciones.

- Susceptibilidad a Movimiento de Laderas.

- Deslizamientos.

- Mapa de Suelos.

- Propuesta de puntos para el Acelerógrafo, entre otros. 
Figura 1. Mapa de Ubicación de la Zona de Estudio

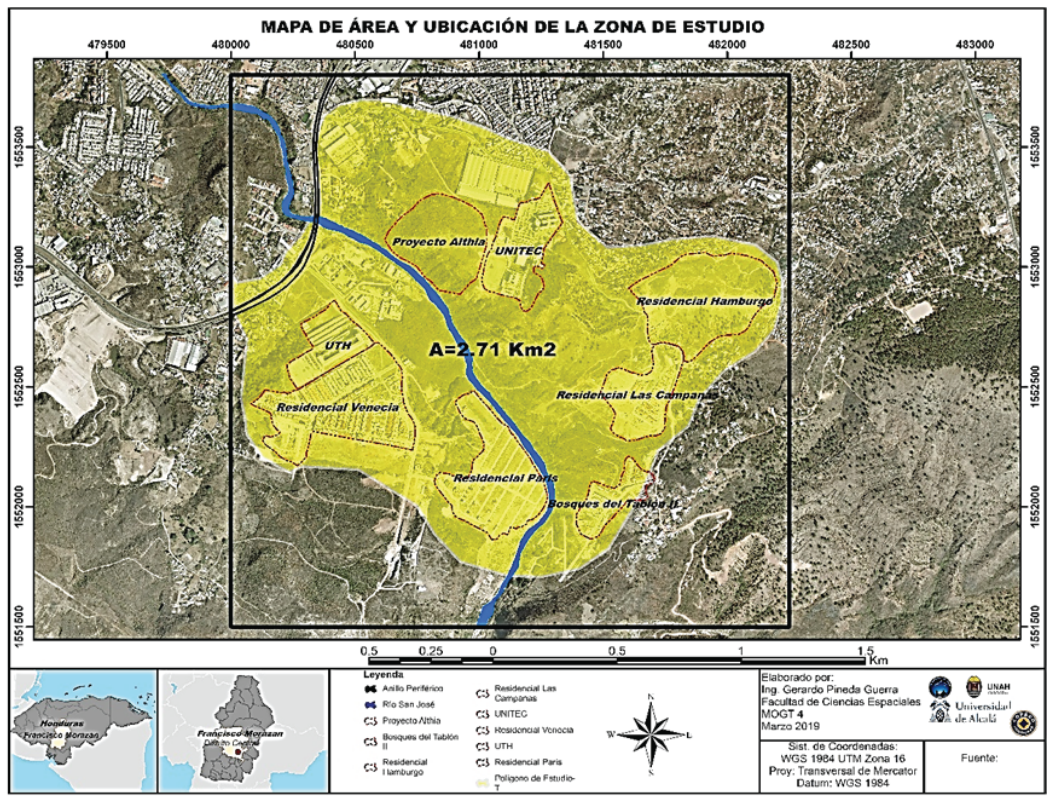

Fuente: Elaboración propia

Figura 2. Mapa de Amenazas Sísmicas de Honduras, Información Nacional

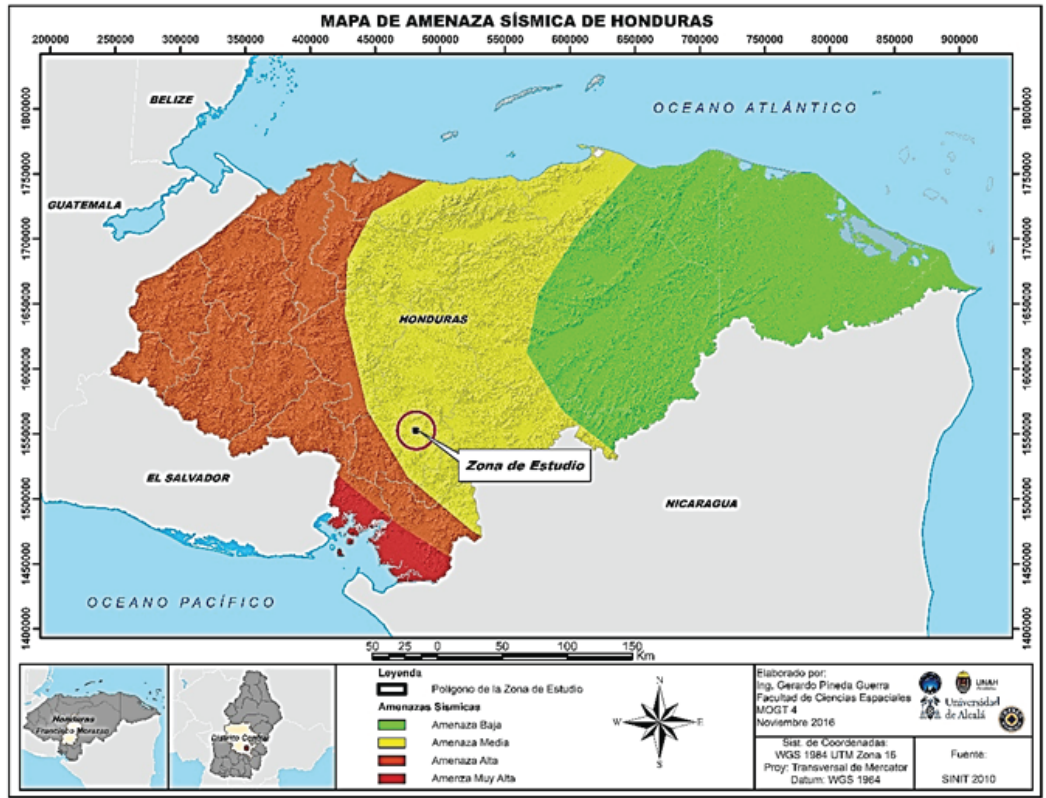

Fuente: Elaboración propia 


\section{Levantamiento Topográfico}

Fue necesario realizar un levantamiento topográfico con estación total para generar curvas de nivel a cada 5 metros de distancia, saber elevaciones y realizar el Modelo de Elevación Digital.

\section{Estudio Geológico a Detalle}

Se visitó la zona de investigación y se realizó una inspección de las rocas, recolección de muestra, también se corroboró con los mapas temáticos realizados las estructuras y fallas geológicas que existen en el sitio de estudio.

\section{Estudios Geofísicos}

- Refracción Sísmica

Uno de los estudios geofísicos que se realizó en la investigación es el ensayo de Refracción Sísmica, este se adapta muy bien para trabajos de Ingeniería Civil, ya que puede medir preliminarmente el perfil del suelo y roca, espesores de recubrimiento, profundidad de nivel freático, realización de estudios hidrológicos, etc.

Equipo usado:

Sismógrafo DoReMi, los elementos que componen el sistema son los geófonos, cable para conexiones y un disco de aluminio.

\section{Figura 3. Equipo y golpeo del plato para obtener datos de Refracción Sísmica}

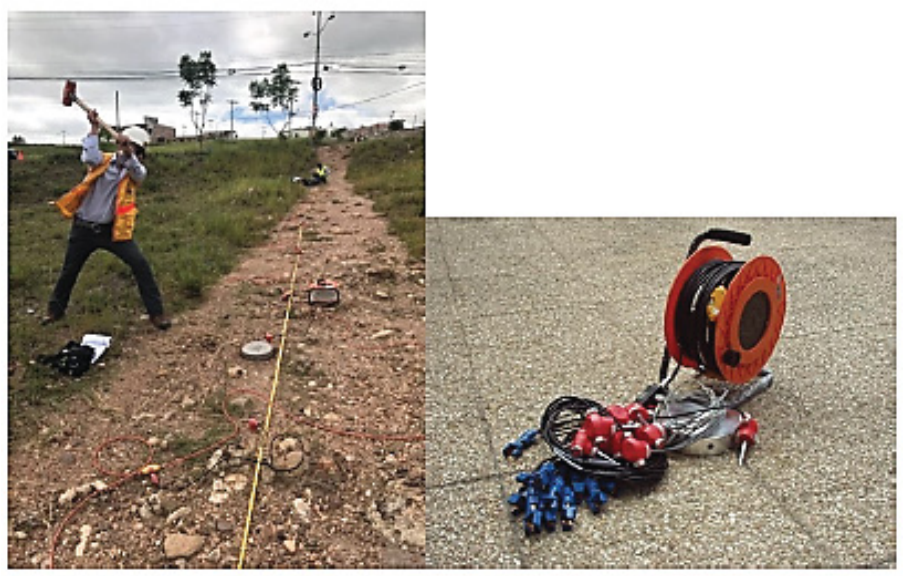


- Resistividad Eléctrica.

Las medidas de resistividad eléctrica del subsuelo son habituales en las prospecciones geofísicas. Su finalidad es detectar y localizar cuerpos y estructuras geológicas basándose en su contraste resistivo. El equipo utilizado en esta investigación fue el Terrameter modelo LS de la casa comercial ABEM.

Para la interpretación de datos se utilizan tablas o gráficos de resistividades típicas, apoyado de información geológica y geotécnica de la zona de estudio.

\section{Figura 4. Ensayo de Resistividad Eléctrica}

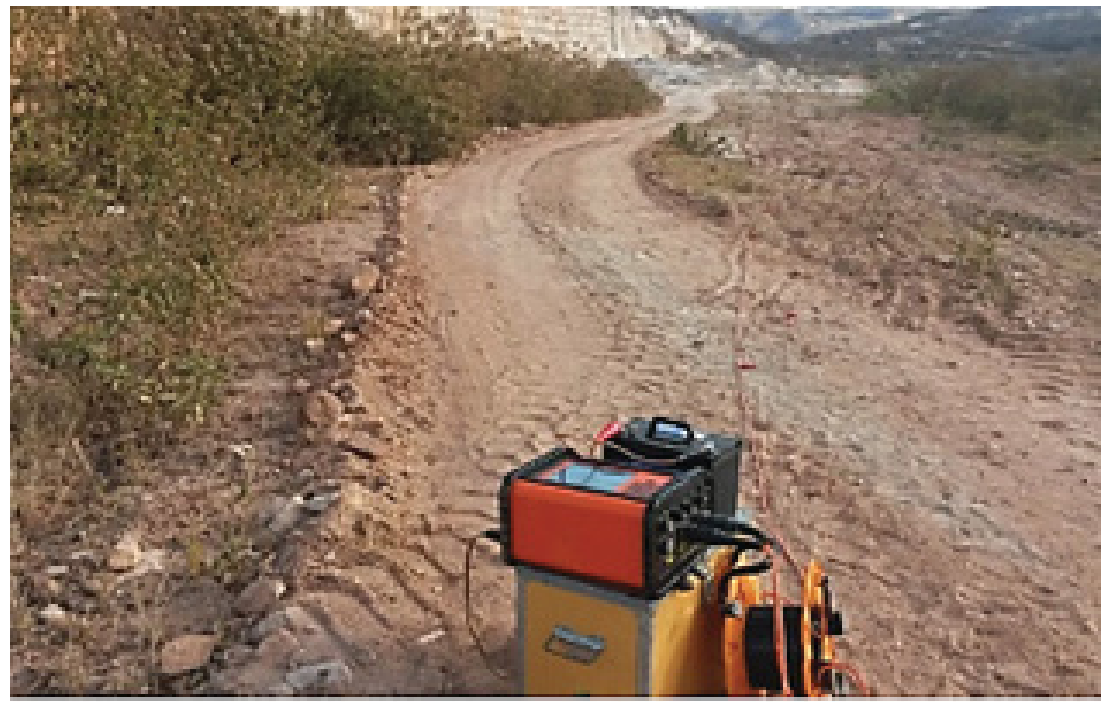

- Tecnica H/N, (Nakamura 1989 y 2000)

Para el estudio se utilizó el acelerógrafo modelo Basalt, serie Rock, de la casa comercial Kinemetrics. Este se coloca sobre el suelo, orientándolo hacia el norte geográfico y asegurando nivelarlo con ayuda del ojo de buey. Este graba durante 5 minutos activándolo manualmente.

Para el procesamiento de los datos se utilizó el programa Geopsy 2.7.0, con el cual se calculan los espectros de Fourier y las Funciones de Transferencia Empírica. En el gráfico se obtiene la amplitud $\mathrm{H} / \mathrm{V}$ en el eje $\mathrm{Y}$, y en el eje $\mathrm{X}$ la Frecuencia del suelo medida en Hertz. ( $T=1 / F)$. 


\section{Figura 5. Toma de puntos con el Acelerógrafo}

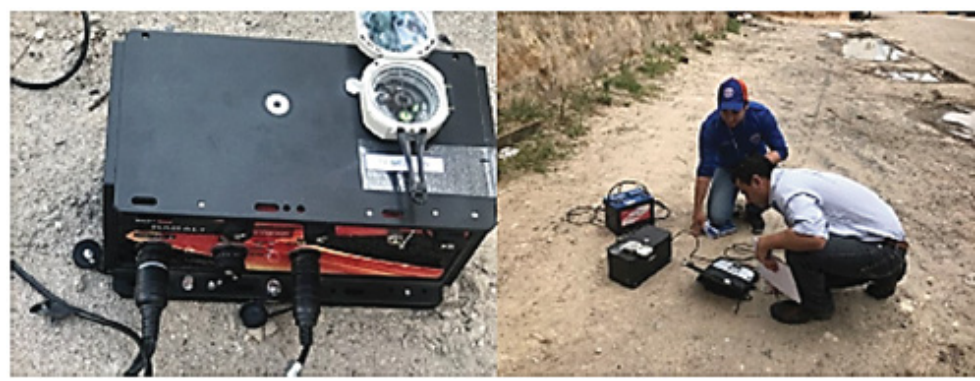

Figura 6. Gráfico representativo obtenido por el programa Geopsy

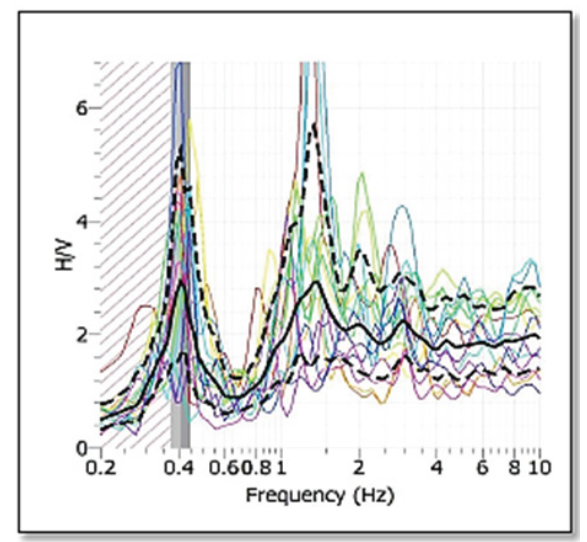

- Posibles errores en los datos o resultados obtenidos en el estudio.

Del análisis del acelerograma, es posible obtener los espectros de respuesta del terreno, los cuales nos proporcionan el período natural de vibración del suelo, debido a que en esta investigación la toma de puntos fue uno en cada punto de interés y se realizó en un intervalo de tiempo de 5 minutos por punto, es posible que la certeza del dato sea mejorada tomando lo que dicen los expertos, se requiere tomar 3 tomas en cada punto y en un intervalo de 5 minutos cada uno, siempre y cuando los puntos estén cercanos uno de otro.

Es necesario realizar pruebas de laboratorios del suelo para tener más certeza en la composición del mismo, así como la realización de perforaciones con recuperación de testigos de rocas para saber a cabalidad como están compuestos los estratos de roca de la zona de estudio, la utilización de estos métodos requiere de recursos importantes y es por esto que en esta investigación no se pudieron realizar. 
El procedimiento de estudio de microzonificación puede variar por diferentes motivos, algunos de estos pudieran ser la experiencia de realizarlos, el dinero con el que se cuenta para lograr el objetivo o el método que se emplee para realizarlo; sea cual sea el método que se utilice los datos obtenidos deben de ser muy similares.

\title{
ANÁLISIS Y DISCUCIÓN DE LOS RESULTADOS
}

\author{
Geología a Detalle.
}

Figura 7. Mapa de la Geología a Detalle de la Zona de Estudio.

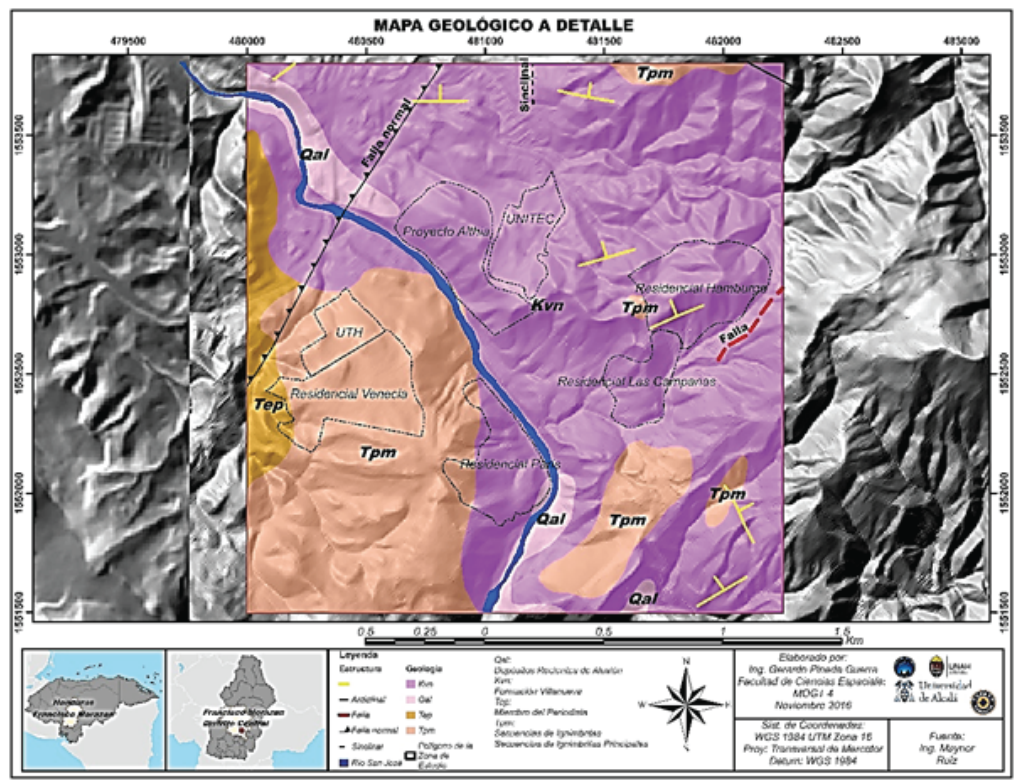

La Zona de estudio presenta un accidente tectónico que marca la división entre dos formaciones geológicas (Grupo Valle de Ángeles y Grupo Padre Miguel), dentro de estas dos formaciones existen dos miembros geológicos: Miembro el Periodista y la Formación Villa Nueva donde se origina un contacto entre ellas. La formación geológica más antigua que se distingue en la zona son las rocas del grupo Padre Miguel, sobre ella se encuentran los Basaltos Cuaternarios.

Del estudio geológico a detalle se obtuvo como resultado una estratigrafía analizada desde la más antigua a las más jóvenes unidades estratigráficas, con lo anterior se 
conoció que la geología del área de estudio presenta una Formación Villa Nueva (Kvn), constituida por estratos siliciclásticos de grano grueso, conglomerados de cuarzo y clastos de rocas (metamórficas, volcánicas y calizas), areniscas de color rojo claro y hasta café claro y algunas tobas volcánicas, Grupo Padre Miguel (Tpm), constituido por una secuencia de ignimbritas principales de tobas riolíticas, dacíticas y andesíticas de varios colores. Algunas rocas sedimentarias de clastos volcánicos y tobas bien estratificadas, El Miembro del Periodista (Tep), constituido por sedimentos volcaniclásticos de areniscas, grava, limonita y Los Depósitos Recientes (Qal), Constituida por depósitos de aluvión, se correlaciona con depósitos de arena, grava, guijarros de terraza y abanicos aluviales.

En resumen, el mapa temático que se presenta en la figura 7, presenta las Fallas Normales, los Pliegues, el rumbo y echado de las capas, las fallas, y la geología Kvn, Tpm, Tep y Qal antes descrita.

\section{Estudio de Refracción Sísmica}

Los datos recolectados se analizaron empleando el método de refracción sísmica. El método de Prospección Sísmica de Refracción, se basa en el registro de los frentes de onda, generados por una fuente artificial de energía, leyendo en los registros los tiempos de los primeros arribos (first breaks).

Como resultado se obtuvieron gráficos de este tipo en donde el eje "Y" es la velocidad de onda en $\mathrm{m} / \mathrm{s}$ y el eje "X" es la distancia en $\mathrm{m}$ :

\section{Figura 8. Perfil del Estudio de Refracción Sísmica realizado en Residencial Venecia.}

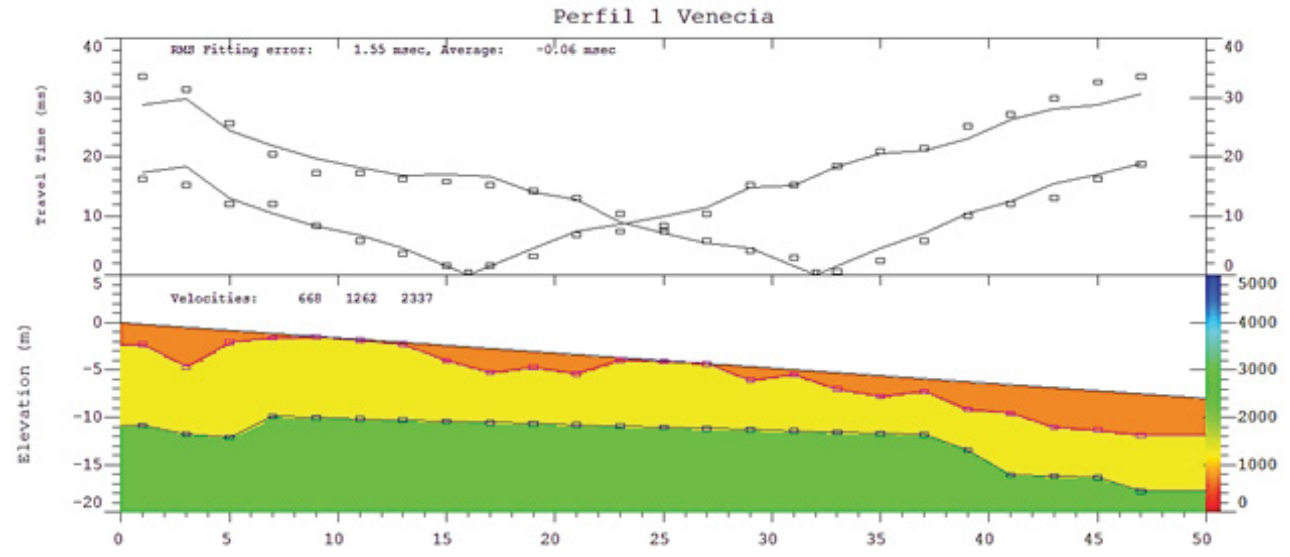


Tabla 1. Resumen de resultados obtenidos del ensayo de Refracción Sísmica.

\begin{tabular}{|c|c|c|c|c|}
\hline $\begin{array}{l}\text { Perfil o Línea } \\
\text { de Refracción }\end{array}$ & $\begin{array}{l}\text { Velocidad de } \\
\text { Onda } P,(\mathrm{~m} / \mathrm{s})\end{array}$ & $\begin{array}{l}\text { Espesor de } \\
\text { la capa }(m)\end{array}$ & Tipo de Suelo & Ubicación \\
\hline \multirow{3}{*}{$\begin{array}{l}\text { Perfil No. 1, } \\
\text { Residencial } \\
\text { Venecia }\end{array}$} & 668 & 1 a 4 & $\begin{array}{l}\text { suelo y materiales sueltos } \\
\text { muy poco consolidados }\end{array}$ & \multirow{2}{*}{ Figura 1, Ubicación del sondeo de Refracción Sísmica, Res. Venecia. } \\
\hline & 1262 & 11 a 13 & $\begin{array}{l}\text { Mezcla de materiales de } \\
\text { depósitos aluviales. }\end{array}$ & \\
\hline & 2337 & 13 a 20 & $\begin{array}{l}\text { roca meteorizada, o } \\
\text { depósitos aluviales con } \\
\text { fragmentos de roca de } \\
\text { mayor tamaño }\end{array}$ & \\
\hline \multirow{3}{*}{$\begin{array}{l}\text { Perfil No. 2, } \\
\text { Residencial } \\
\text { Paris }\end{array}$} & 646 & 1 a 4 & $\begin{array}{l}\text { suelo y materiales sueltos } \\
\text { muy poco consolidados, un } \\
\text { suelo muy denso y roca } \\
\text { suave }\end{array}$ & \multirow[t]{3}{*}{ Figura 2, Ubicación del sondeo Residencial Paris. } \\
\hline & 1297 & 9 a 10 & $\begin{array}{l}\text { mezcla de materiales de } \\
\text { depósitos aluviales, } \\
\text { aproximándose a la roca }\end{array}$ & \\
\hline & 1956 & 10 a 15 & $\begin{array}{l}\text { Roca meteorizada, o } \\
\text { depósitos aluviales con } \\
\text { fragmentos de roca de } \\
\text { mayor tamaño. }\end{array}$ & \\
\hline \multirow{3}{*}{$\begin{array}{l}\text { Perfil No. 3, } \\
\text { Bosques del } \\
\text { Tablón }\end{array}$} & 418 & 1 a 4 & $\begin{array}{c}\text { suelo y materiales } \\
\text { medianamente densos o } \\
\text { medianamente rígidos y } \\
\text { suaves }\end{array}$ & \multirow[t]{3}{*}{$\begin{array}{c}\text { Figura 3, Ubicación del sondeo de refracción Sismica, Bosques del } \\
\text { Tablón. }\end{array}$} \\
\hline & 918 & 4 a 8 & $\begin{array}{l}\text { mezcla de materiales de } \\
\text { depósitos aluviales, } \\
\text { aproximándose a la roca en } \\
\text { donde también podemos } \\
\text { encontrar suelos rígidos }\end{array}$ & \\
\hline & 1585 & 8 a 10 & $\begin{array}{l}\text { Roca meteorizada, o } \\
\text { depósitos aluviales con } \\
\text { fragmentos de roca de } \\
\text { mayor tamaño. }\end{array}$ & \\
\hline \multirow{3}{*}{$\begin{array}{l}\text { Perfil No. } 4 \text {, } \\
\text { Residencial } \\
\text { Las Campanas }\end{array}$} & 365 & 1 a 5 & $\begin{array}{l}\text { suelo y materiales } \\
\text { medianamente densas o } \\
\text { medianamente rígidas y } \\
\text { suaves, también se } \\
\text { presenta un material } \\
\text { arcilloso }\end{array}$ & \multirow[t]{3}{*}{ Figura 4, Ubicación del sondeo Residencial Las Campanas. } \\
\hline & 1592 & 6 a 9 & $\begin{array}{l}\text { mezcla de materiales de } \\
\text { depósitos aluviales, } \\
\text { aproximándose a la roca en } \\
\text { donde también podemos } \\
\text { encontrar suelos rígidos }\end{array}$ & \\
\hline & 2984 & 9 a 20 & $\begin{array}{l}\text { Roca meteorizada, o } \\
\text { depósitos aluviales con } \\
\text { fragmentos de roca de } \\
\text { mayor tamaño. }\end{array}$ & \\
\hline
\end{tabular}




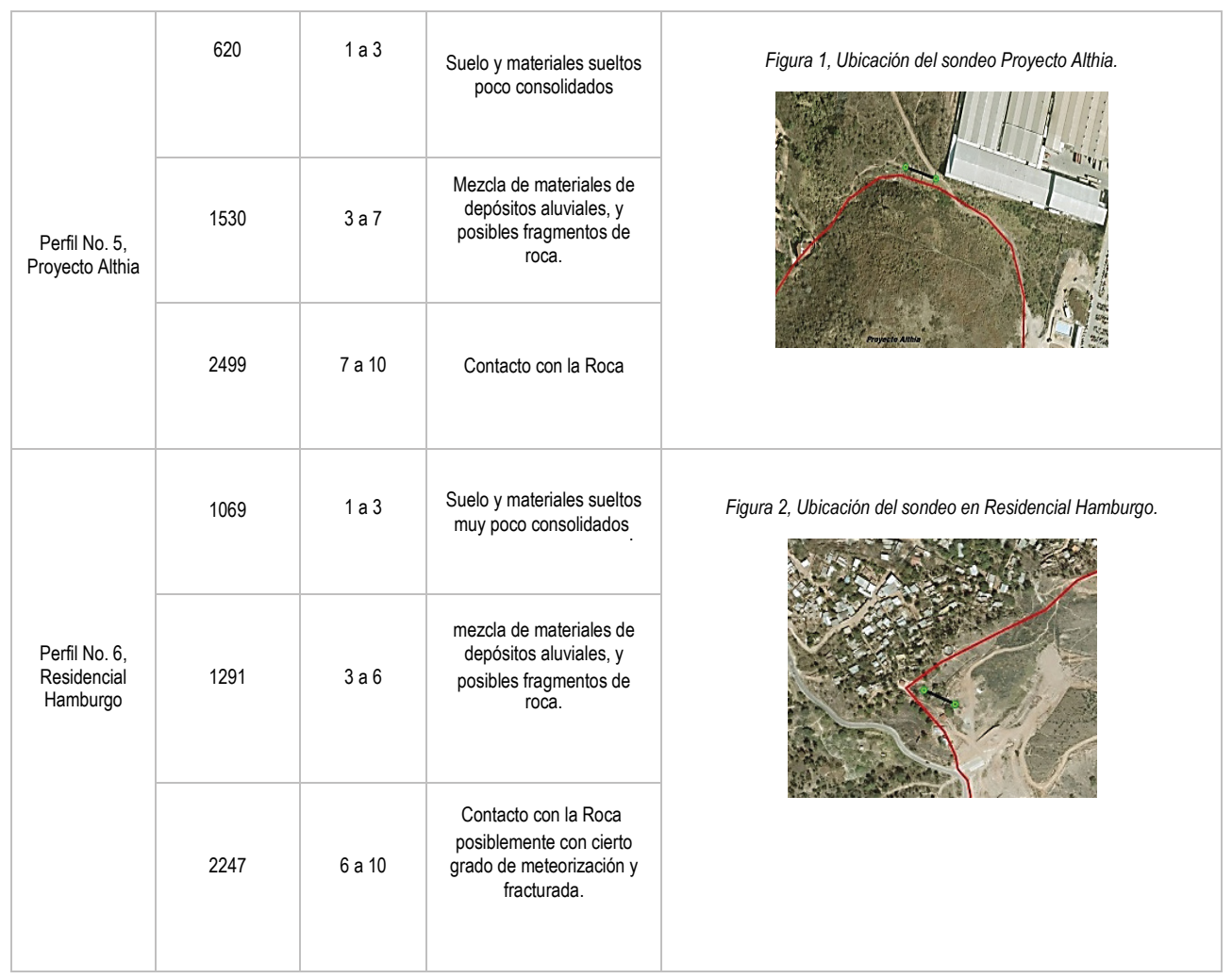

\section{Estudio de Resistividad Eléctrica}

En un Sondeo Eléctrico Vertical (SEV) se obtiene una variación de la resistividad en relación a la profundidad. Los electrodos se ubican de tal manera que se mida la resistividad en un punto $(0)$, que corresponde al centro del arreglo, y se van separando gradualmente para realizar mediciones a diferentes profundidades, cada vez más grandes, hasta llegar a tener una separación igual o preferiblemente mayor, entre los electrodos de corriente $A B$, al doble a la profundidad que se desea para el estudio geológico, (Estrada, Prospección Geoeléctrica para Ingenieros, 2015).

Debido a que la profundidad siempre estará referida al punto centro a partir de este estudio es posible determinar los espesores de diferentes capas presentes en el subsuelo, en dicho punto. 
Figura 15. Sondeo Eléctrico Vertical.

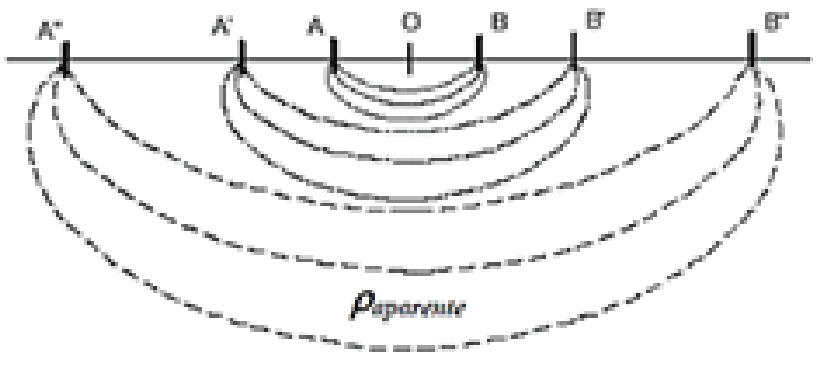

\section{Estudio de Resistividad Eléctrica}

La resistividad $p$ (rho) de un medio es la propiedad física que determina la capacidad de este medio para dejar pasar la corriente eléctrica. La conductividad $\sigma$ es el inverso de la resistividad y se expresa en ohm/m (ohmios por metro).

Figura 16. Perfil del Sondeo de Resistividad Eléctrica realizado en Residencial Venecia.

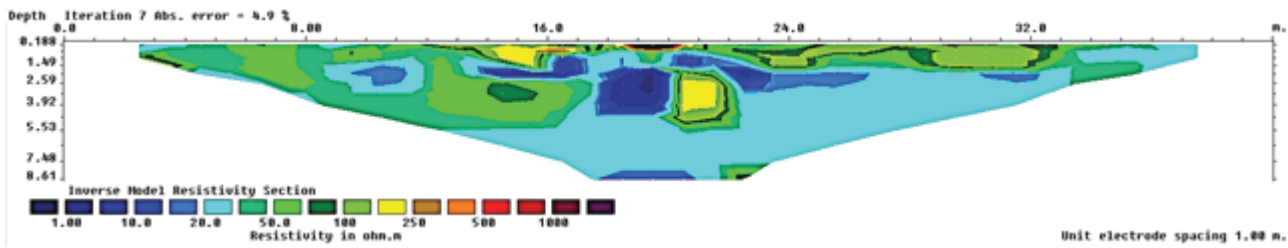

\section{Método de Razón Espectral H/V, (Método de Nakamura).}

En la zona de estudio se lograron tomar 8 puntos que se ubicaron desde el anillo periférico frente a la Universidad Tecnológica de Honduras (UTH), dentro de la misma Universidad, en la Residencial Venecia, en Altos de Venecia, en la Residencial Paris, y en un terreno de la Bloquera GUFER.

A continuación, se presentan ejemplos de los gráficos obtenidos para la zona en estudio, así como la tabla resumen de resultados que finalmente se obtiene para generar la propuesta de Microzonificación Sísmica a través de Sistemas de Información Geográfica. 


\section{Tabla 2. Resumen de resultados del ensayo de Resistividad Eléctrica.}

\begin{tabular}{|c|c|c|}
\hline $\begin{array}{l}\text { Perfil y } \\
\text { ubicación del } \\
\text { sondeo }\end{array}$ & $\begin{array}{l}\text { Profundidad } \\
(\mathrm{m})\end{array}$ & Resultado del sondeo \\
\hline $\begin{array}{l}\text { Perfil No. 1, } \\
\text { Altos de } \\
\text { Venecia }\end{array}$ & 8.61 & $\begin{array}{l}\text { Las capas menos resistivas de colores azul celeste a verde (1-100 } \Omega \text {-m), Corresponden al miembro } \\
\text { mediano de la secuencia ignimbrítica. Estas son capas relativamente blandas y consisten en tobas } \\
\text { rayadas, arcillas, que están en muchas áreas de Tegucigalpa. No se observa contacto con el } \\
\text { basamento rocoso }\end{array}$ \\
\hline $\begin{array}{l}\text { Perfil No. 2, } \\
\text { Altos de } \\
\text { Venecia }\end{array}$ & 17.20 & $\begin{array}{l}\text { Estructuras sedimentarias, como canales paleo, laminación, imbricación de graduación normal en } \\
\text { clastos, y arcillas. No se observa contacto con el basamento rocoso. }\end{array}$ \\
\hline $\begin{array}{l}\text { Perfil No.1, } \\
\text { Venecia II } \\
\text { Etapa }\end{array}$ & 63 & $\begin{array}{l}\text { Secuencia ignimbrítica inferior. En este miembro se encuentran tobas pómez riolíticas y también } \\
\text { dacíticas/andesíticas en una profundidad de } 1 \text { a } 20 \mathrm{~m} \text {, a aproximadamente después de los } 20 \mathrm{~m} \text { de } \\
\text { profundidad se encuentran rocas sedimentarias. }\end{array}$ \\
\hline $\begin{array}{l}\text { Perfil No.2, } \\
\text { Venecia II } \\
\text { Etapa }\end{array}$ & 63 & Capas blandas, tobas rayadas, arcillas, este tipo de geología es susceptible a deslizamientos. \\
\hline $\begin{array}{l}\text { Perfil No. 1, } \\
\text { Residencial } \\
\quad \text { Paris }\end{array}$ & 55 & $\begin{array}{l}\text { (200-500 } \Omega \text {-m) son capas rojas de la formación basal del Grupo Valle de Ángeles. Estas capas } \\
\text { principalmente de cuarzos conglomerados de matriz arenosa. Algunos cuarzos son muy duros y otros } \\
\text { son más blandos (15-50 } \Omega \text {-m) en los límites estas capas se vuelven resbaladizas. }\end{array}$ \\
\hline $\begin{array}{l}\text { Perfil No. 2, } \\
\text { Residencial } \\
\text { Paris }\end{array}$ & 60 & $\begin{array}{l}\text { Contacto entre dos formaciones geológicas. Produce infiltración de agua y deslizamientos, se puede } \\
\text { observar que de } 0 \text { a } 10 \mathrm{~m} \text { de altura son rellenos. }\end{array}$ \\
\hline $\begin{array}{l}\text { Perfil No. 3, } \\
\text { Residencial } \\
\quad \text { Paris }\end{array}$ & 50 & $\begin{array}{l}\text { Capas rojas, arenosas y arcillosas, en ciertos tramos hay bolones de roca, se puede observar que de } 0 \\
\text { a } 10 \mathrm{~m} \text { de altura son rellenos. }\end{array}$ \\
\hline $\begin{array}{l}\text { Perfil No. } 4 \\
\text { Residencial } \\
\quad \text { Paris }\end{array}$ & 70 & $\begin{array}{l}\text { Material de capas rojas, arenosas y arcillosas, también conglomerados blandos, y depósitos aluviales } \\
\text { esto debido a que esta zona está ubicada en las cercanías del Río San José. }\end{array}$ \\
\hline $\begin{array}{l}\text { Perfil No. 5, } \\
\text { Residencial } \\
\quad \text { Paris }\end{array}$ & 70 & $\begin{array}{l}\text { Material de capas rojas, arenosas y arcillosas, también conglomerados blandos, y depósitos aluviales } \\
\text { esto debido a que esta zona está ubicada en las cercanías del Río San José. }\end{array}$ \\
\hline $\begin{array}{l}\text { Perfil No. 6, } \\
\text { Residencial } \\
\quad \text { Paris }\end{array}$ & 70 & $\begin{array}{l}\text { Material arcilloso, y depósitos aluviales que han formado rellenos en el suelo, esto debido a que esta } \\
\text { zona está ubicada en las cercanías del Río San José. En estas zonas los terrenos son susceptibles a } \\
\text { desplazamientos y deslizamientos del mismo material de relleno. }\end{array}$ \\
\hline $\begin{array}{l}\text { Perfil No. } 7 \\
\text { Residencial } \\
\quad \text { Paris }\end{array}$ & 140 & $\begin{array}{l}\text { Material arcilloso, y depósitos aluviales que han formado rellenos en el suelo, esto debido a que esta } \\
\text { zona está ubicada en las cercanías del Río San José. En estas zonas los terrenos son susceptibles a } \\
\text { desplazamientos y deslizamientos del mismo material de relleno. }\end{array}$ \\
\hline $\begin{array}{l}\text { Perfil No. } 1 \text { y } \\
\text { 2, Residencial } \\
\text { El Tablón II }\end{array}$ & 40 & $\begin{array}{l}\text { En ambos perfiles se representan las resistividades bajas ( } 0 \text { a } 50 \Omega \text {-m) que estos materiales podrían } \\
\text { corresponder a sedimentos arcillosos no consolidados. Depósitos de material fino erosionado de los } \\
\text { conglomerados de la formación Villa Nueva. Resistividades de color azul entre }(0 \text { a } 10 \Omega \text {-m), } \\
\text { corresponderían a inclusión de agua en el suelo de relleno producto de infiltraciones de agua lluvia. } \\
\text { Esta sería la causante de que muros y paredes se asienten y causen las fisuras. } \\
\text { Estratos con mayor resistividad }(50-250 \Omega \text {-m), constituirían depósitos coluviones matriz soportados. } \\
\text { Conglomerados de clastos finos en una matriz arcillosa. } \\
\text { Capas con mayor resistividad (<250 } \Omega \text {-m) representarían depósitos de coluviones de conglomerados } \\
\text { con clastos de material granular en matriz arcillosa. }\end{array}$ \\
\hline
\end{tabular}


Figura 17. Gráficos representativos de las frecuencias obtenidas en la zona de estudio.
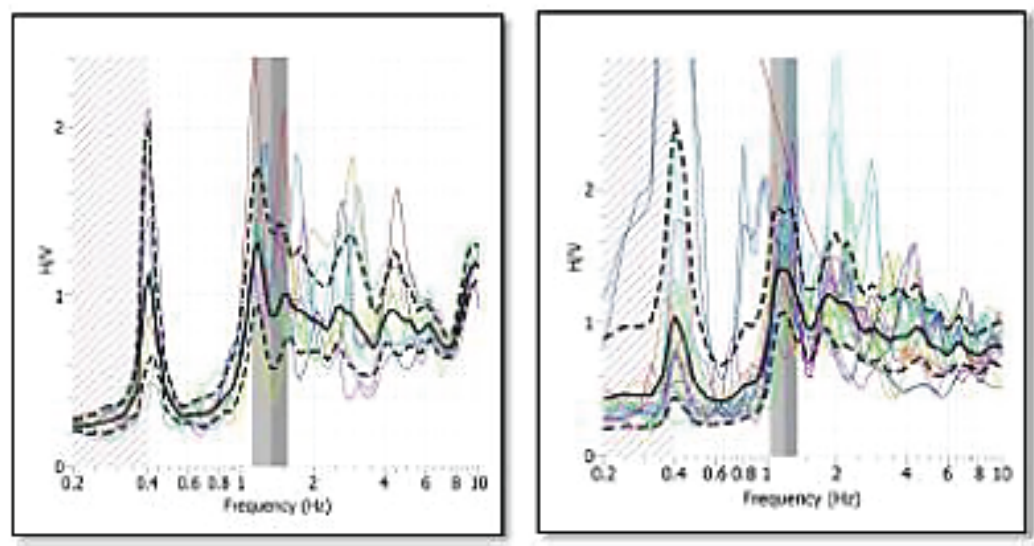

Para el análisis de estos resultados obtenidos se consideró que en efecto puede haber períodos de 0.05, 0.1 a 1.0, 2.0 segundos, generados por tránsito vehicular, operación de centros fabriles, el viento que hace oscilar árboles entre otros factores, pero los períodos obtenidos se relacionan con el período dominante para microtremores.

Entre las principales aplicaciones del estudio de microtremores tenemos:

- Obtención de los periodos dominantes del suelo.

- Detección de la estructura subterránea (la vibración del periodo es consistente con el perfil geológico).

- Obtención de perfiles de velocidades de onda.

El análisis propone dos microzonas definidas entre las Frecuencias $(F)$ que van desde $0.56 \mathrm{~Hz}$ a $0.80 \mathrm{~Hz}$ para la primera microzona con un rango de periodos (To) que va de 1.25 a 1.79 segundos, y que podría ser clasificada como suelo tipo IV: Suelo aluvial de depósitos deltaicos blandos, suelo superficial, limos, con espesores de 30 metros o más; según la clasificación de suelos basado en microtremores propuesta dentro del código de contracción japonés. La segunda zona con Frecuencias de arriba de 1.25 hasta los $2.30 \mathrm{~Hz}$ que implican periodos en el orden de $0.43 \mathrm{a}$ 0.80 segundos, clasificada como un tipo de suelo II: gravas arenosas, arcillas arenosas duras y otros suelos de tipo aluvial gravosos, con espesores de 5 metros o más. 
Figura 18. Mapa de Frecuencias en Hertz obtenidas mediante registros de ruido ambiental.

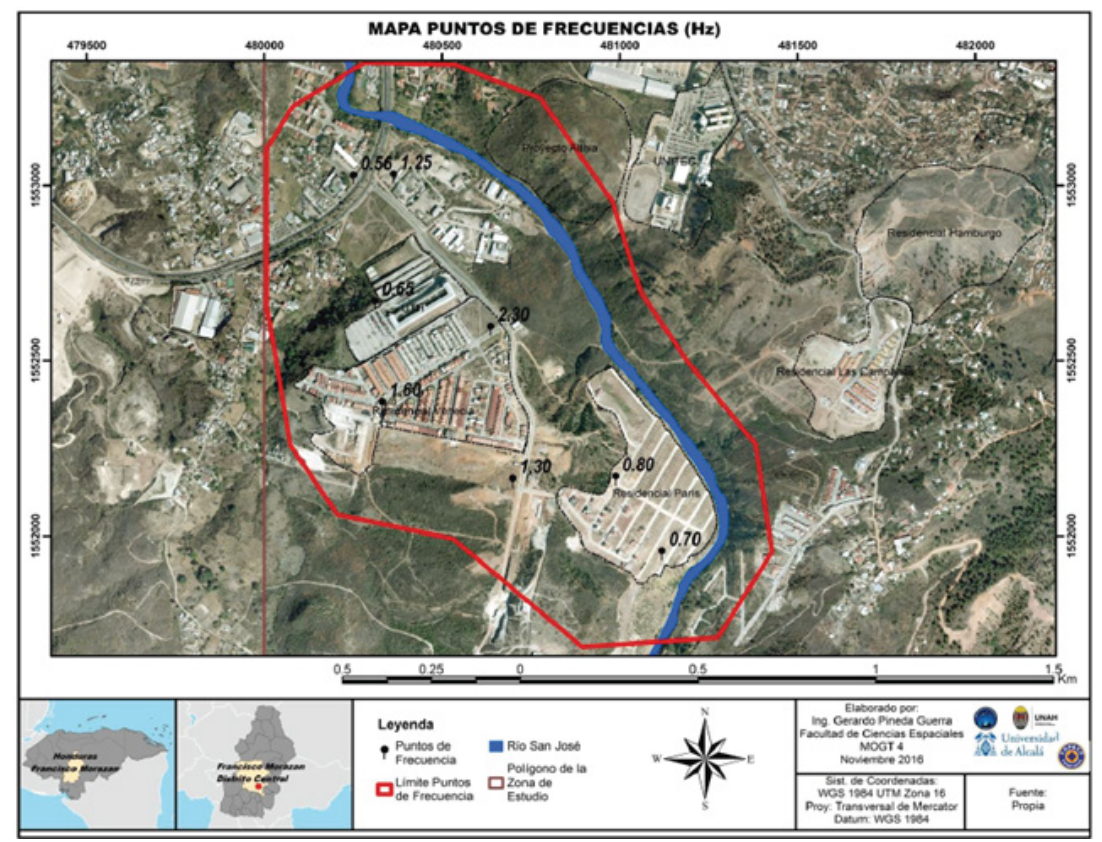

La Tabla siguiente resume los resultados de todas las mediciones realizadas para esta zona y que generan la propuesta del mapa de microzonificación. Es de notar que de los 8 registros tomados, 5 de ellos presentan amplitudes (A) mayores a 1.20, llegando en algunos puntos hasta 2.40 veces, esto indica que toda el área podría presentar Efecto de Sitio en el momento de ocurrir un sismo.

Tabla 3. Mapa Resumen de resultados para la zona de estudio (Frecuencia en Hertz, Amplitud en veces y Período fundamental en segundos).

\begin{tabular}{|l|l|l|l|l|l|l|}
\hline PUNTO & LATITUD & LONGITUD & $\mathbf{F}(\mathrm{Hz})$ & A (veces) & To $(\mathbf{s e g})$ & EF \\
\hline 1 & 1553021 & 480251 & 0.56 & 0.32 & 1.79 & $\mathrm{SI}$ \\
\hline 2 & 1552663 & 480312 & 0.65 & 0.48 & 1.54 & $\mathrm{SI}$ \\
\hline 3 & 1552590 & 480637 & 2.30 & 2.40 & 0.43 & $\mathrm{SI}$ \\
\hline 4 & 1552375 & 480332 & 1.60 & 0.88 & 0.63 & $\mathrm{SI}$ \\
\hline 5 & 1552156 & 480699 & 1.30 & 1.50 & 0.77 & $\mathrm{SI}$ \\
\hline 6 & 1552162 & 480988 & 0.80 & 1.20 & 1.25 & $\mathrm{SI}$ \\
\hline 7 & 1551949 & 481119 & 0.70 & 1.97 & 1.43 & $\mathrm{SI}$ \\
\hline 8 & 1553024 & 480363 & 1.25 & 1.20 & 0.80 & $\mathrm{SI}$ \\
\hline
\end{tabular}


Figura 19. Mapa de Amplitudes Relativas obtenidas mediante registros de ruido ambiental.

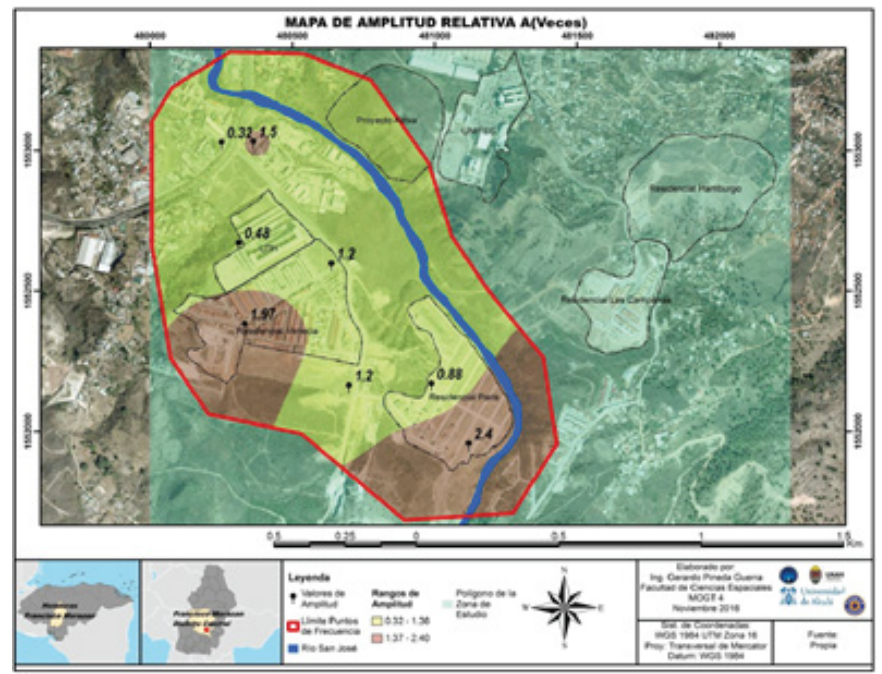

Las amplitudes encontradas para el sitio estudiado, indican que es una zona propensa a presentar Efecto de Sitio, de modo que las señales sísmicas pueden verse amplificadas en este suelo y el daño estructural puede ser significativo en toda la zona de acuerdo a los periodos de vibración estimados en este estudio.

Figura 20. Propuesta de Mapa de Microzonificación Sísmica para la Zona de Estudio.

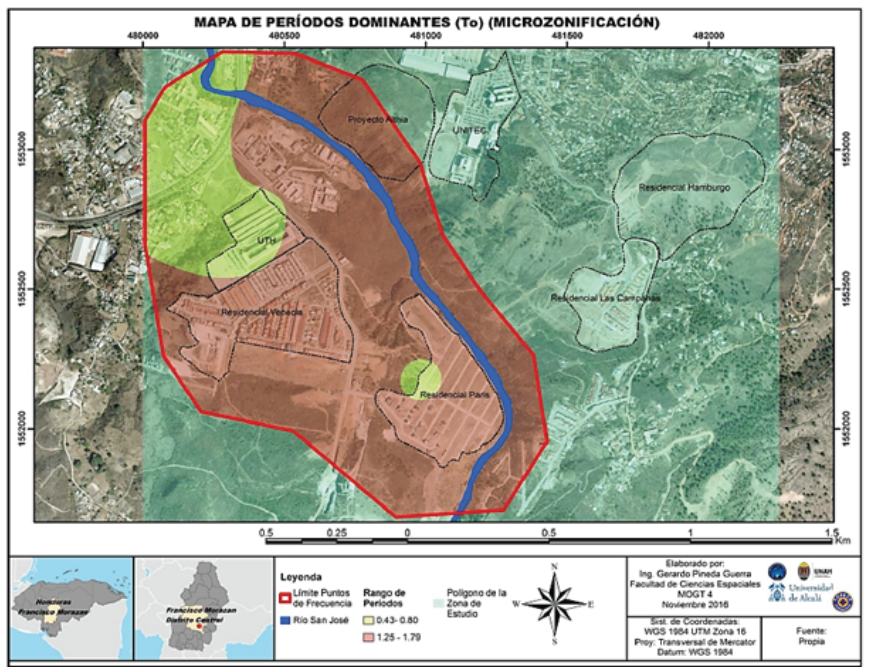

Se proponen dos microzonas definidas como se observan en la tabla 2 
Tabla 4. Microzonas propuestas.

\begin{tabular}{|l|l|}
\hline Zona & To (segundos) \\
\hline I & $1.25-1.79$ \\
\hline II & $0.43-0.80$ \\
\hline
\end{tabular}

Para la zona uno las edificaciones en riesgo son las que poseen una altura promedio correspondiente arriba de los diez pisos. En la zona dos las edificaciones de 3 a 4 pisos.

\section{CONCLUSIONES}

La Estructura Geológica y Geomorfológica del suelo de la zona de estudio es compleja, donde la heterogeneidad del material ha permitido el desarrollo de procesos de movimientos en masa, principalmente en las rocas de origen volcánico, también las rocas sedimentarias existentes, presentan mejor calidad, desde el punto geotécnico, sin embargo, dado los ángulos de buzamiento, $>45^{\circ}$, se han desarrollado procesos de caídas de bloques y volcamiento de bloques.

Después de realizar el levantamiento topográfico y el modelo de elevación digital del terreno, se concluye que las alteraciones hidrotermales e intemperismo químico son los fenómenos que han alterado los materiales geológicos, confiriéndoles pobre calidad geotécnica, sumado a las fuertes pendiente de la zona, han desarrollado la inestabilidad de laderas.

Siguiendo la Variable Social y Demográfica se concluye que el daño de varias edificaciones y familias afectadas se dio por el mal control que llevó la Alcaldía Municipal en dar los permisos de construcción, ya que para solicitar dicho permiso se deben de cumplir con requerimientos de diseño sismo resistente así como diferentes estudios geotécnicos a detalle.

Los resultados arrojados de Frecuencias (0.56-2.30 Hz), Amplitudes (0.32-2.40 veces) y Períodos fundamentales (0.43-1.79 segundos), acompañados de todos los estudios geológicos y geofísicos realizados muestran que en el terreno de estudio se presentan zonas con geologías similares en donde predominan los suelos blandos por lo que se concluye que si se produciría el Efecto de Sitio. 
Se realizaron un total 8 mediciones en el suelo del lado oeste de la zona de estudio, tomando puntos cerca de algunos edificios y tratando de aprovechar lo más que dio el tiempo y el equipo para abarcar el área de estudio, encontrándose 8 puntos con efecto de sitio en los rangos de frecuencia medidos, debido a que para profundidades bajas las frecuencias son altas y van a ser mayores de 0.2 , valores menores de frecuencia de 0.2 normalmente es basamento rocoso.

Para el mapa de Microzonificación Sísmica del presente estudio se proponen dos tipos de Zonas, Zona I que posee períodos (To) de 1.25-1.79 segundos y la Zona II que posee períodos de $0.43-0.80$ segundos, para la Zona I las edificaciones en riesgo son las que poseen una altura promedio correspondiente a los diez pisos, en la Zona Il las edificaciones de 3 a 4 pisos.

\section{AGRADECIMIENTOS}

A todos mis asesores por dedicar el tiempo para ayudarme con mi investigación y a la UNAH-DICU-DICYP por haber financiado la beca de investigación.

\section{REFERENCIAS BIBLIOGRÁFICAS}

Nakamura, Y. (1989). A method for dynamic characteristics estimation of subsurface using microtremor on the ground surface. QR Railway Tech. Res. Inst., 30(1), 25-33.

Nakamura, Y. (2000, January). Clear identification of fundamental idea of Nakamura's technique and its applications. In Proceedings of the 12th world conference on earthquake engineering (Vol. 2656). New Zealand: Auckland.

Luis A. Estrada. (2015). Prospección Geoeléctrica para Ingenieros. Recuperado de https://catedras.facet.unt.edu.ar/geofisica/wp-content/uploads/sites/4/2014/02/Geoelectrica-para Ingenieros.pdf 